\section{Neue Rufnummern der Pollenflugvorhersage}

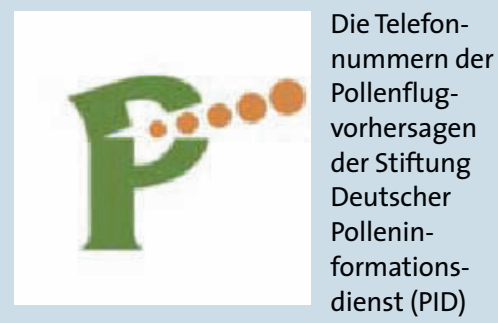

haben sich zum 1. Januar 2006 geändert. Die Regionalansagen für die einzelnen Bundesländer sind jetzt zum Preis von 0,62 Cent pro Minute (aus dem deutschen Festnetz) unter folgenden Nummern zu erreichen:

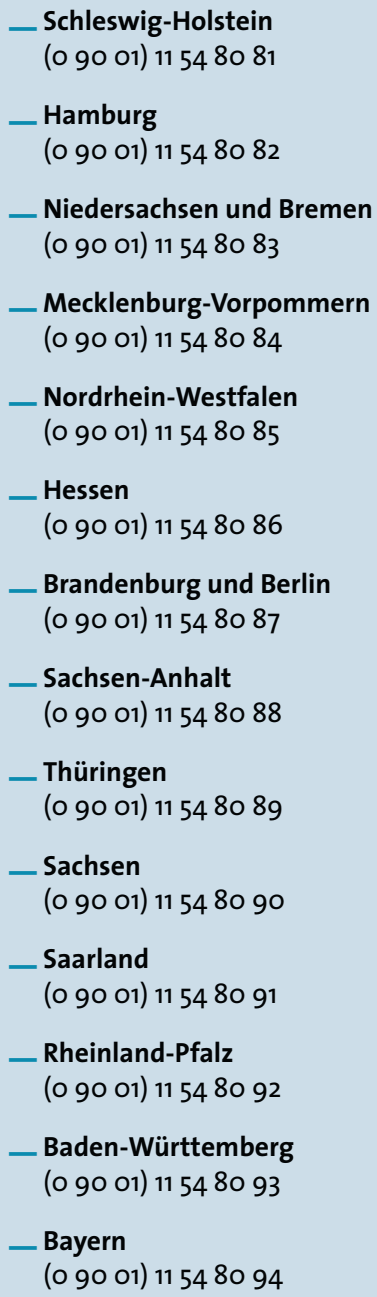

Aktuelle Informationen sind auch über die Homepage des Polleninformationsdienstes www.pollenstiftung.de abrufbar. Auf europäischer Ebene informiert das Europäische Polleninformationssystem www.polleninfo.org über das Pollenfluggeschehen.

\section{Neues Siegel für Nahrungsmittelallergiker}

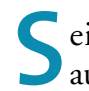
auch in Deutschland die EU-Richtlinie 200/89 in Kraft, die eine strengere Deklaration der Bestandteile von Nahrungsmitteln vorsieht. Insbesondere sind die zwölf häufigsten Auslöser von Lebensmittelallergien und -unverträglichkeiten grundsätzlich in der Zutatenliste aufzuführen. Der Berliner Stiftung für Allergieforschung (ECARF) der Hautklinik der Charité geht das noch nicht weit genug. Sie hat ein eigenes Qualitätssiegel entwickelt, das Allergikern beim Einkauf noch mehr Sicherheit geben soll.

Hersteller dürfen ihre Produkte nur mit dem Siegel auszeichnen, wenn sie regelmäßige Kontrollen des Endproduktes durchführen lassen. Nur wenn die Konzentrationen der zwölf deklarierungspflichtigen Allergene unter der Schwelle von $10 \mathrm{mg} / \mathrm{kg}$ liegen, müssen sie nicht genannt werden. Darüber hinaus werden die Kontrollen auch auf seltenere Nahrungsmittelallergene ausgedehnt. „Das Allergiesiegel überprüft damit nicht nur die gesetzlich geforderte Nennung von Inhaltsstoffen, sondern auch unbeabsichtigte Allergenspuren und eine zusätzliche Deklaration weiterer häufiger Allergene, wenn sie in der Rezeptur des Nahrungsmittels verweneit dem 25. November 2005 ist

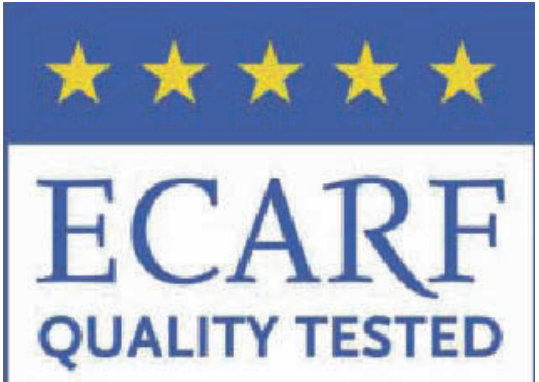

Informationen zu Allergien: www.allergy-foundation.info

European Centre for Allergy Research Foundation

\section{Dieses Siegel will die Europäische Stiftung für Allergieforschung ab sofort Lebensmittelprodukten verleihen, deren Hersteller besondere Rücksicht auf Nahrungsmittelallergiker nehmen.}

det wurden“, erklärt Prof. Dr. Torsten Zuberbier, Leiter der ECARF.

Die regelmäßigen Kontrollen der Endprodukte übernimmt ein unabhängiges Institut. Das Siegel wird kostenfrei auf Antrag der Nahrungsmittelhersteller vergeben. Auf den Internetseiten des ECARF www.allergy-foundation.info sind ausführliche Informationen über das neue ECARF-Qualitätssiegel hinterlegt.

ECARF

\section{Gratisexemplar für DGAKI-Mitglieder}

Im Jahr 2004 hat das Aktionsbündnis Allergieprävention ( $a b[a] p)$ erstmals Leitlinien zur Allergieprävention vorgelegt. Die Empfehlungen, deren Kurzfassung auch im Allergo Journal erschienen ist (Allergo J 2004; 13: 252-60), wurden nach einem hohen methodischen Standard entwickelt und spiegeln die vorhandene Evidenz zur Allergieprävention in Deutschland wider.

Seit 2005 liegen die Leitlinien auch als Langfassung in Buchform vor. Da das Buch mit Unterstützung des Bundesministeriums für Gesundheit und Soziale Sicherung gedruckt worden ist, kann Mitgliedern der Deutschen Gesellschaft für Allergologie und klinische Immunologie ein Freiexemplar zur Verfügung gestellt werden. Es kann schriftlich direkt bei den Autoren der Leitlinie abgerufen werden.

\begin{tabular}{l}
\hline Prof. Dr. Torsten Schäfer \\
Dr. Cathleen Borowski \\
Institut für Sozialmedizin, Beckergrube 43-47, 23552 Lübeck \\
Fax: (o4 51) 7992539 \\
E-Mail: kristina.berger@sozmed.uni-luebeck.de
\end{tabular}

